\title{
Combining syntactic patterns and Wikipedia's hierarchy of hyperlinks to extract meronym relations
}

\author{
Debela Tesfaye, Michael Zock and Solomon Teferra \\ ITPHD PROGRAM, Addis Ababa University, Addis Ababa, Ethiopia \\ LIF-CNRS, 163 Avenue de Luminy, 13288 Marseille, France \\ Addis Ababa University, Addis Ababa, Ethiopia \\ dabookoo@yahoo.com, michael.zock@lif.univ-mrs.fr, solomon_teferra_7@yahoo.com
}

\begin{abstract}
We present here two methods for extraction o, meronymic relation : (a) the first one relies solely on syntactic information. Unlike other approaches based on simple patterns, we determine their optimal combination to extract word pairs linked via a given semantic relation; (b) the second approach consists in combining syntactic patterns with the semantic information extracted from the Wikipedia hyperlink hierarchy $(W H H)$ of the constituent words. By comparing our work with SemEval 2007 (Task 4 test set) and WordNet (WN) we found that our system clearly outperforms its competitors.
\end{abstract}

\section{Introduction}

The attempt to discover automatically semantic relations $(S R)$ between words, or word pairs has attracted a number of researchers during the last decade which is understandable given the number of applications needing this kind of information. Question Answering, Information Retrieval and Text Summarization being examples in case (Turney and Littman, 2005; Girju et al., 2005).

SRs extraction approaches can be categorized on the basis of the kind of information used. For example, one can rely on syntactic patterns or semantic features of the constituent words. One may as well combine these two approaches.

The method using only syntactic information relies on the extraction of word-level, phrase-level, or sentence-level syntactic information. This approach has been introduced by Hearst (1992) who showed that by using a small set of lexicosyntactic patterns ( $L S P)$ one could extract with high precision hypernym noun pairs. Similar methods have been used since then by (Auger and Barriere, 2008; Marshman and L'Homme, 2006). These authors reported results of high precision for some relations, for example hyponymy, noting poor recall which was low. Furthermore, the performance of this approach varies considerably depending on the type of relation considered (Ravichandran and Hovy, 2002, Girju et al., 2005.

An alternative to the syntactic approach is a method relying on the semantics features of a pair of words. Most researchers using this approach (Alicia, 2007; Hendrickx et.al, 2007) rely on information extracted from lexical resources like WN (Fellbaum, 1998). Alas, this method works only for languages having a resource equivalent to $W N$. Yet, even WN may pose a proble because of its low coverage across domains (tennis problem).

Hybrid approaches consist in the combination of syntactic patterns with the semantic features of the constituent words (Claudio, 2007; Girju et.al 2005). They tend to yield better results. However, their reliance on $W N$ make them amenable to the same criticism as the ones just mentioned concerning $W N$. More recently Wikipedia based similarity measures have been proposed (Strube, et.al, 2006; Gabrilovich, and Markovitch, 2007). While this strategy produces excellent results, few attempts have been made to extract SRS (Nakayama et. al, 2007; Yulan et, al , 2007).

In this paper we propose two approaches to extract meronymic relations. In the first case we rely on the patterns learned from LSPs. Previous syntactic approaches aimed at finding stand-alone, unambiguous $L S P$, for instance $X$ such as $Y$, in order to extract a semantic relation like hyponymy. Yet, such unambiguous, stand-alone LSPs are very rare and yield low performance. Instead of using $L S P s$ individually, which are often ambiguous, we try to combine them in such a way that they com- 
plete each other. For instance, the ambiguity of the pattern " $N N_{1}$ make of $\mathrm{NN}_{2}$ " can be reduced via the pattern " $N N_{2}$ to make $N N_{l}$ " in order to extract meronymy. $N N_{1}$ and $N N_{2}$ can stand for any pair of words. The second approach consists in disambiguating the word pairs extracted by LSPS via the information identified from the Wikipedia pages of the respective words.

Our contributions are twofold. First, we propose a novel technique for extracting and combining LSPs in order to extract SRs. Second, we propose an approach for disambiguating the syntactic patterns (say meronymic patterns like $\mathrm{NN}_{1}$-has$\mathrm{NN}_{2}$ ) by building a hyperlink-hierarchy based on Wikipedia pages.

\section{Our Approach in more detail}

Previous work relies on unambiguous, stand alone LSPs to extract SRs. While this approach allows for high precision, it has been criticized for its low accuracy and its variability in terms of the SRs to be extracted. Not all SRs are equally well 'identified'. One of the main challenges and motivations for LSP mining lies in the disambiguation of $L S P$ to allow for the extraction of SRs. To achieve this, we propose two methods:

- Determine an optimal combination of LSPs to represent the relation at hand (section 2.1).

- Combining LSPS with the semantic features of the constituent words extracted from the Wikipedia hyperlink-hierarchy (section 2.2).

\subsection{Combination of syntactic patterns for relation extraction (CoSP-FRe)}

The use of individual LSP for the extraction of word pairs linked via a given $S R$ tends to produce poor results (Girju et al., 2005; Hearst, 1998). One reason for this lies in the fact that the majority of word pairs are linked via polysemous LSPs (Girju et.al , 2005). Hence, these patterns cannot be used alone, as they are ambiguous. At the same time they cannot be ignored as they have the potential to provide good clues concerning certain SRs. This being so we suggest to assign weights to the $L S P S$ according to their relevance for a specific $S R$, and to optimally combine such weighted patterns for extracting word pairs linked via the SR at hand.
In order to determine the optimal combination of $L S P S$ likely to extract $S R s$, we have harvested all $L S P S$ encoding the relation at hand. We assigned weights to the patterns according to their relevance for the given $S R s$, and finally filtered the best combination of LSPS.

In order to extract such patterns linking word pairs via a certain $S R$, we selected seed-word pairs representative of the relation at hand. In order to balance the word pairs we followed standard taxonomies to group the relations and selected samples from each group (see Section 3.1.1). Sentences containing the word pairs were extracted and then identified their dependency structure. We identified dependency structure linking the word pairs using the shortest path (ex. nsubj(have, aircraft) and dobj(have, engines) from the sentence aircrafts have engine). Having replaced the words by $N N_{1}$ (whole) and $N N_{2}$ (part) we obtained patterns like $N N_{1}$ have $N N_{2}$. We finally counted the frequency of the LSPS and ordered them according to their frequency and considering the top 50.

Determination of the optimal combination of LSPs encoding a given $\boldsymbol{S R}$. To determine the optimal combination of LSPs, we identyfied the discrimination value $(D V)$ for each pattern. The $D V$ is a numerical value signaling the relevancy of a given $L S P$ with respect to a given SR. We applied the following steps in order to identify the $D V$ and to determine the optimal combination of the LSPs:

Step 1: For each extracted $L S P$, we extracted more connected word pairs from Wikipedia. We defined regular expression matching sentences linking word pairs via the LSPS and built then word pairs in a LSPs matrix (Matrix 1). Table 1 below shows sample word pairs connected by the patterns $N N_{l}$ has $N N_{2}$ and $N N_{2}$ of $N N_{l}$. Next, we labeled the extracted word pairs with the SR type and built a matrix of word pairs by a specific $S R$ type (Matrix 2). In Table 2 the word pairs from matrix 1 are labeled with their respective type of $S R$. We relied on WN to automatically label the word pairs. Starting with the first sense of the words occurring in $W N$, we traverse the hierarchies and identify the $S R S$ encoded by the word pairs. Using the information from Matrix 1 and 2, we built a matrix of SRs to LSPS (Matrix 3). Table 3 shows sample Matrix 3. The rows of the matrix represent the SR type, while columns represent the 
$L S P s^{\prime}$ encoding. The cells are populated by the number of word pairs linked by the $L S P$ encoding the $S R$. The $D V$ of $L S P$ for a given $S R$ is given by the following formula:

$$
D V=\frac{F P R}{F P} * \log \left(\frac{T N R}{T R E}\right)
$$

$F P$ represents the total number of word pairs connected by the $L S P$ (from Matrix 1). FPR stands for the number of word pairs connected by the given $S R$ (from Matrix 2), while $T N R$ and TRE represent respectively the total number of $S R S$ (from Matrix 3) and the total number of SRs encoded by the pattern (from Matrix 3).

\begin{tabular}{|l|l}
\hline Word Pairs & LSP \\
\hline Car Engine & $\mathrm{NN}_{1}$ has $\mathrm{NN}_{2}$ \\
\cline { 1 - 1 } Girl Car & $\mathrm{NN}_{1}$ has $\mathrm{NN}_{2}$ \\
\cline { 1 - 1 } Door Car & $\mathrm{NN}_{2}$ of $\mathrm{NN}_{1}$ \\
\cline { 1 - 1 } Aircraft Engine & $\mathrm{NN}_{1}{ }^{\prime} \mathrm{NN}_{2}$ \\
\multicolumn{2}{|c}{ Table 1: Sample Matrix 1. }
\end{tabular}

\begin{tabular}{|l|l}
\hline Word Pairs & SR Type \\
\hline Car Engine & Meronymy \\
\cline { 1 - 1 } Girl Car & Possession \\
\cline { 1 - 1 } Door Car & Meronymy \\
\cline { 1 - 1 } Aircraft Engine & Meronymy \\
\multicolumn{2}{|c|}{ Table 2: Sample Matrix 2. }
\end{tabular}

\begin{tabular}{|c|c|c|c|}
\hline SR Type & $\mathbf{N N}_{l}$ has $\boldsymbol{N N}_{2}$ & $\mathrm{NN}_{2}$ of $N N_{1}$ & $\mathrm{NN}_{1}{ }^{\prime} \mathrm{NN}_{2}$ \\
\hline Meronymy & 1 & 1 & 1 \\
\hline Possession & 1 & 0 & 0 \\
\hline
\end{tabular}

Step 2: Identify the optimal combination of $L S P$ to represent a given relation. First, we build a matrix combining LSPs encoding the respective $S R s$ (Matrix 4) from matrix 3. The LSPs in Matrix 3 are combined until no other combination is possible. The cells of the Matrix 4 are populated by the number of word pairs linked via the respective combination of LSPs. Next we calculated the discrimination value $(D V-g)$ for the combined $L S P$ s, the $D V-g$ being calculated for each combination of $L S P$ corresponding to a given SR. We then selected the combination of LSPs with maximum $D V-g$ for each SR. The $D V-g$ for the combined LSPs corresponding to a given SR is given by the following formula:

$D V-g=\frac{F P R-g}{F P-g} * \log \left(\frac{T N R}{T R E-g}\right)$

$F P$ - $g$ expresses the total number of word pairs connected by the group of patterns. It is determined by taking the intersection of word pairs connected via the combined LSPs (from Matrix 4), where $F P R-g$ represents the number of word pairs connected by the combined $L S P S$ for a given $S R$. This value is determined by taking the intersection of positive word pairs connected by the combined $L S P$ for a given $S R$ (from Matrix 4). Finally, TNR and TRE represent respectively the total number of $S R s$ (from Matrix 4) and the total number of SRs encoded by the combination of the $L S P$.

\begin{tabular}{|c|c|c|}
\hline SR Type & $N N_{l}$ has $N N_{2}+N N_{2}$ of $N N_{1}$ & $N N_{2}$ of $N N_{1+} N N_{1}{ }^{\prime} N N_{2}$ \\
\hline Meronymy & 2 & 2 \\
\hline Possession & 0 & 0 \\
\hline
\end{tabular}

As can be seen from table 3, the pattern " $N N_{l}$ has $\mathrm{NN}_{2}$ " when used independently encodes both a meronymic and a non-meronymic word pair. From table 4 above there are two meronymic word pairs linked by the combination of patterns " $N N_{1}$ has $N N_{2}+N N_{2}$ of $N N_{1}$ " while there are no nonmeronymic word pairs. Hence the non-meronymic word pair retrieved via the pattern " $\mathrm{NN}_{1}$ has $\mathrm{NN}_{2}$ " is filtered out as a result of having combined it with the pattern " $N N_{2}$ of $N N_{l}$ ".

\subsection{Wikipedia hyperlink hierarchies for $S R$ extraction (WHH-Fsre): the case of meronymy extraction}

We used here the hyperlink-hierarchies built on the basis of a selected set of sentences of Wikipedia pages containing the respective word pairs in order to disambiguate LSPS encoding them. The basic motivations behind this approach are as follows:

1. Words linked to the Wikipedia page title (WPT) via $L S P$ encoding $S R$ are more reliable than word pairs linked in arbitrary sentences.

2. Word pairs encoding a given SR are not always directly connected via $L S P S$. SRs encoded by a given word pair can also be encoded by their respective higher/lower order conceptual terms. For instance, the following two sentences "germ is an embryo of seed" and "grain is a seed" yield relations like hyponymy (germ, embryo, and grain, seed), meronymy (embryo, seed, and germ, grain), the latter (germ, grain) being inferred via the relation of their higher order terms (embryo and seed).

The candidate meronymic word pairs extracted via meronymic LSPS are further refined by using 
the patterns learned from their conceptual hierarchies built on the basis of semantic links, namely, 'hypernymic-link' (HL), and the 'meronymic-link' $(M L)$. We extracted the hyperlinks connected to the Wikipedia pages of the respective meronymic candidates by using hypernymic and meronymic $L S P$. The hyperlink hierarchies were built by considering only important sentences ( 1 and 2 below) from the Wikipedia pages of the pair of terms: (1) definition sentences and (2) sentences linking hyperlinks to the WPT using meronymic LSPS. Since the meronymic $L S P$ vary according to the nature of the arguments, the patterns used to extract hyperlinks for building the hierarchies were learned by taking the nature of the meronymic relations into account (section 2.1). The definition sentences are used to extract hypernymic-hyperlink ${ }^{1}$, and the sentences linking hyperlinks to the $W P T$ using meronymic LSPS are used to extract meronymic-hyperlink ${ }^{2}$. Using the hierarchy constructed for the candidate word pairs, this approach determines whether the pairs are meronyms or not based on the following assumptions:

(a) The hyperlink hierarchies of hierarchical meronymys constructed form their respective $H L$ have a common ancestor in the hierarchy. Figure 1 shows the component-Integral meronyms 'car engine' sharing the parent 'machine' in their hyperlinkhierarchy constructed from their respective Wikipedia page definitions.

(b) The hyperlink hierarchies of both hierarchical and non-hierarchical-meronyms constructed from their respective $M L$ and/or $H L$ converge along the path in the hierarchy.

Extraction of the hyperlinks. To extract the hyperlinks, we performed the following operations: Step 1: For simple meronymic pairs we identified the respective Wikipedia pages aligning the word pairs with the WPT based on the overlap of the

${ }^{1}$ The hypernymic-hyperlink is a word defining a term via its higher-order concept, providing in addition a hyperlink to other Wikipedia pages for further reading. The hypernymichyperlinks are underlined on figure 1 .

${ }^{2}$ Meronymic-hyperlink is a word describing a term using its whole concept and providing a hyperlink to other Wikipedia pages for further reading. surface word form. The word pairs were selected based on standard categories used for describing meronymic taxonomy (Winston et al. 1987, see also section 3.1.1). We first cleaned Wikipedia articles and extracted Wikipedia definitions and sentences linking WPT with hyperlinks using meronymic LSPS.

Step 2: Annotations. We manually annotated both kinds of sentences using two kinds of information: WPT and the hyperlinks. The hyperlink either links the term to its meronyms or hypernyms.

Step 3: Extract LSPS linking the WPT with the hyperlinks. We assigned $D V$ (section 2.1) for the patterns and considered the most frequent LSPS. The hyperlinks broadly fall in either of two categories: (a) hypernymic-hyperlink. They are extracted by the patterns linking the tuple (hyperlink, wpt), for instance, is-a (hyperlink, Wikipedia page title) as in the example (b,c); (b) meronymic-hyperlinks. They are extracted via LSPs linking the tuple (hyperlink, wpt), for instance, made-from (hyperlink, wpt).

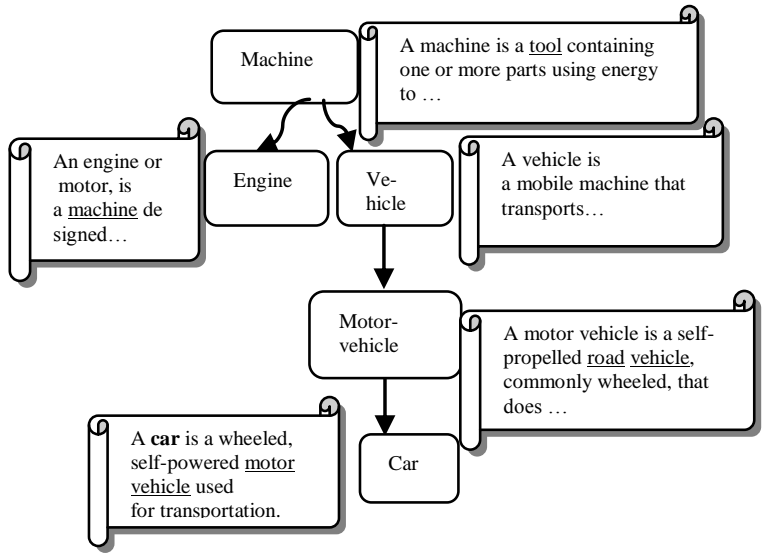

Fig. 1. Wikipedia definitions and the resulting hypernymic-hyperlink hierarchies for the meronyms 'car engine'

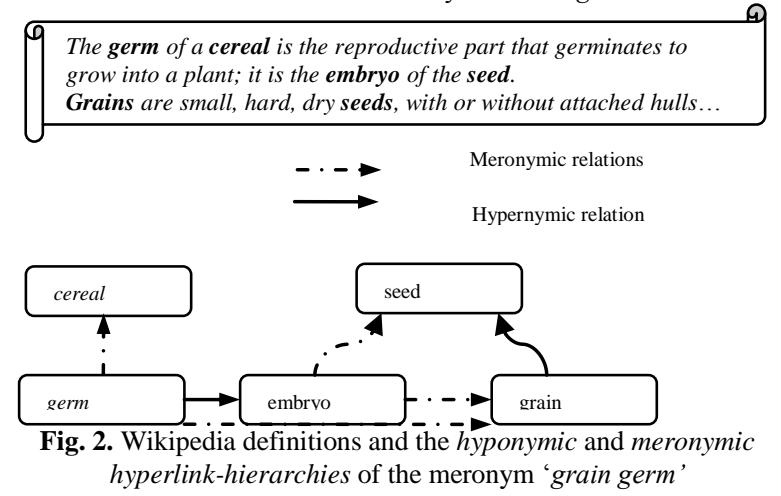

Constructing the hierarchy. For a given pair of terms, we identified the respective Wikipedia 
pages, by aligning the pairs with the wpt and by using word form overlap to extract their associated initial hypernymic and meronymic hyperlinks $\left(h l_{i}\right)$ based on the patterns learned in step 2.2.1. We further identified the respective Wikipedia pages for the hypernymic and meronymic-hyperlink $\left(\mathrm{hl}_{\mathrm{i}}\right)$ identified before and extracted the associated hypernymic and meronymic hyperlinks $\left(\mathrm{hl}_{\mathrm{i}+1}\right)$. Next we connected $\left(h l_{i}\right)$ with $\left(h l_{i+1}\right)$ to form a hierarchy (hypernyms are connected to each other and to meronyms and vice versa). The hyperlinks are extracted until the hierarchies converge, or until the hypernymic-hierarchy reaches seven layers (most word pairs converge earlier than that).

Decide on the meronymic status of words. The hypernymic or meronymic-hyperlink of one of the words of the pair is searched in the hierarchy of the other, and if this link occurs we consider the word pairs as meronyms. Figure 2 shows that the meronymic word pair 'germ grain' converges at 'seed' in the hierarchies built from their respective Wikipedia pages.

\section{Experiment}

To show the validity of our line of reasoning we carried out three experiments:

I. Extract the optimal combination of LSPS encoding meronymic relation only.

II. Evaluate $C o S P-F R e$ for meronymy extraction.

III. Evaluate WHH-Fre for extracting meronymy.

\subsection{Extract the optimal combination of LSPs encoding meronymy}

Training data set. Two sets of data are required: (a) the initial meronymic word pairs used to train our system (b) the corpus from which the LSPS were selected. To select the representative list of meronymic pairs, we used a standard taxonomy. Indeed, several scholars have proposed taxonomies of meronyms (Winston et al., 1987; Pribbenow, 1995; Keet \& Artale, 2008). We followed Winston's classical proposal:

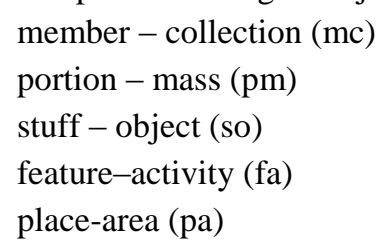

For the training we used the part-whole training set of SemEval-2007 task 4 (Girju et al. 2007) .

Experimental setup. To determine the optimal combination of LSPs encoding meronyms we identified LSPS encoding meronymy according to the procedures described in section 2.1. Since most of these patterns are rare we considered only those with a frequency of 100 and above. For individual $L S P$ extraction, we identified the $D V s$ associated with the meronymic relation by using the formula 1 followed by the $D V-g s$ for every combination of $L S P s$ by using the second formula. The combined $L S P S$ are sorted based on their $D V$. Finally we selected the LSP with the highest $D V$ as representatives of the respective meronymic types.

\begin{tabular}{|c|l|l|}
\hline Sno & Pattern & DV-g \\
\hline 1 & $\begin{array}{l}\mathrm{NN}_{1} \text { make of } \mathrm{NN}_{2}+\mathrm{NN}_{2} \text { to make } \\
\mathrm{NN}_{1}+\mathrm{NN}_{2} \text { used } \mathrm{NN}_{1}+\mathrm{NN}_{1} \mathrm{NN}_{2}\end{array}$ & $83.6 \%$ \\
\hline 2 & $\mathrm{NN}_{1}$ make from $\mathrm{NN}_{2}+\mathrm{NN}_{2}$ to make & $81 \%$ \\
& $\mathrm{NN}_{1}+\mathrm{NN}_{2}$ used $\mathrm{NN}_{1}+\mathrm{NN}_{1} \mathrm{NN}_{2}$ & \\
\hline
\end{tabular}

Table 5. Part of the optimal combination of patterns for staff object meronymic relations

As can be seen from Table 5 the $D V-g$ of staff object meronymic relations patterns is 83.6. The discrimination values for the $L S P$ in the group when used individually is below $50 \%$.

Evaluation. The goal is to evaluate the degree of correspondance between the meronyms extracted by CoSP-FRe and WHH-FRe on one hand and the one by human annotators on the other.

Test data set. We used two data sets: (a) the partwhole test set of the SemEval-2007 task 4 (Girju et al. 2007) which contains 72 examples (26 positive and 46 negative) and some meronymic word pairs gleaned from WN.

Comparison with other systems. We have compared our work against three approaches that achieved the best performance on SemEval-2007 task 4, and two other approaches. We categorized these approaches as (a) WN-based: CMU-AT (Alicia, 2007) \& ILK (Hendrickx et.al, 2007), (b) syntactic and (c) hybrid approaches: FBK-IRST (Claudio, 2007) \& Girjus et.al (2005). We used the individual LSPS (ILSP) extracted in Sections 2.1 \& the LSPs extracted by Girju, et.al (2005) as syntactic approach. The LSPS extracted by Girju, et.al (2005) are the subset of the LSPs extracted in Sections 2.1. 
Results. We computed precision, recall and Fmeasures as the performance metric. Precision is defined as the ratio of the number of correct meronyms extracted and by the total number of extracted word pairs. Recall is defined as the ratio between the number of correct meronyms extracted and the total number of meronyms in the test set.

\begin{tabular}{|l|l|l|l|}
\hline Approaches & P & R & F \\
\hline CoSP-FRe & $76 \%$ & $88 \%$ & $81.5 \%$ \\
\hline WHH-FRe & $88 \%$ & $90 \%$ & $88.9 \%$ \\
\hline ILSP & $41.6 \%$ & $87 \%$ & $56.2 \%$ \\
\hline CMU-AT & $57.7 \%$ & $45.5 \%$ & $50.8 \%$ \\
\hline FBK-IRST & $65.5 \%$ & $73.1 \%$ & $69.1 \%$ \\
\hline ILK & $48.4 \%$ & $57.7 \%$ & $52.6 \%$ \\
\hline
\end{tabular}

Table 6. Recall (r), Precision (p) and F-Measure (f) of our approach and related works in the SemEval 2007 test set

We have also extracted meronymic word pairs from random Wikipedia pages of 100 articles and added $85 \%$ of the word pairs encoded in $W N$.

Discussions. The results for both approaches are discussed here below:

CoSP-FRe. The precision of CoSP-FRe is improved over syntactic approach as the ambiguity of the individual LSP's is reduced when patterns are combined. Recall is improved as a result of using ambiguous LSPS for extracting word pairs. This contrasts with all the other syntactic approaches which relied only on unambiguous $L S P$. In our approach, ambiguous $L S P S$ are also used in combination with other LSPS. Hence the coverage is significantly improved.

WHH-FRe. Several kinds of hierarchies were formed. Some of them are made of hypernymic or meronymic links, while others are a combination of both links. WHH-FRe outperforms significantly previous approaches both with respect to recall and precision as it combines two important features. First LSPS are used to extract lists of candidate pairs. Second semantic features of the constituent words extracted from Wikipedia hyperlinkhierarchy is used to further refine. Precision is improved for several reasons: relations encoding $L S P S$ which link hyperlinks and WPT are more reliable than word pairs connected via arbitrary sentences. The features learned from the Wikipedia hyperlink-hierarchy further cleaned the word pairs extracted by LSPS. Recall is also improved since word pairs indirectly linked via their respective higher/lower order hierarchy were also extracted.

\section{Related Works}

\subsection{Syntactic approaches}

The work of (Turney, 2005, 2006; Turney and Littman, 2005; Chklovski and Pantel, 2004) is closely related to our work (CoSP-Fre) as it also relies on the use of the distribution of syntactic patterns. However, their goals, algorithms and tasks are different. The work of (Turney, 2005, 2006; and Turney and Littma, 2005) is aimed at measuring relational similarity and is applied to the classification of word pairs (ex. quart: volume vs mile: distance) while we are aimed at extracting SRs.

\subsection{Hybrid approaches}

The work of Girju et.al (2005) is more related to our WHH-FRe in that they combined LSPS with the semantic analysis of the constituent words to disambiguate the LSPs. They used $W N$ to get the semantics of the constituent words. Alicia (2007) converts word pairs of the positive examples into a semantic graph mapping the pairs to the $W N$ hypernym hierarchy. Claudio (2007) combines information from syntactic processing and semantic information of the constituent words from $W N$. Wikipedia-based approaches mainly focused on the identification of similarity (Nakayama et. al, 2007; Yulan et, al , 2007). Also, there is hardly any recent work concerning the extraction of meronyms. Many researchers are working on the identification of semantic similarity achieving excellent result by using standard datasets (Camacho-Collados, Taher and Navigli, 2015; Taher and Navigli , 2015). Yet, most of this work dates back to 2010 and before.

\section{Conclusions}

We presented here two novel approaches for extracting SRs: CoSP-FRe and WHH-FRe. The strength of CoSP-FRe is its capacity to determine an optimal combination of LSPS in order to extract $S R s$. The approach yielded high precision and recall compared to other syntactic approaches. WHH-FRe perform significantly better than previous approaches both with respect to recall and precision as our approach combines $L S P$ and the lexical semantics of the constituted words gleaned from their respective Wikipedia pages. 


\section{References}

Alain A. and Caroline B. (2008). Pattern-based approaches to semantic relation extraction: A stateof-the-art. Terminology Journal, 14(1):1-19

Alicia T. and Scott E. Fahlman (2007). CMU-AT: Semantic Distance and Background Knowledge for Identifying Semantic Relations. Proceedings of the 4th International Workshop on Semantic Evaluations (SemEval-2007), pages 121-124, Prague.

Chklovski, T., and Pantel, P. (2004). VerbOcean: Mining the Web for fine-grained semantic verb relations. In Proceedings of Conference on Empirical Methods in Natural Language Processing (EMNLP-04). pp. 33-40. Barcelona, Spain.

Claudio G., Alberto L., Daniele P. and Lorenza R. (2007). FBK-IRST: Kernel Methods for Semantic Relation Extraction. Proceedings of the 4th International Workshop on Semantic Evaluations (SemEval-2007), pages 121-124, Prague.

Fellbaum, C. editor. (1998). WordNet: An electronic lexical database and some of its applications. MIT Press.

Gabrilovich, E., Markovitch, S. (2007). Computing semantic relatedness using wikipedia-based explicit semantic analysis. In: International Joint Conference on Artificial Intelligence, pp. 12-20.

Girju R., Moldovan D., Tatu, M. \& Antohe, D. (2005). Automatic discovery of Part-Whole relations. ACM 32(1)

Girju, R., Nakov, P., Nastase, V., Szpakowicz, S., Turney, P., \& Yuret, D. (2007). Semeval- 2007 task 04: Classification of semantic relations between nominals. In Proceedings of the Fourth International Workshop on Semantic Evaluations (SemEval 2007), pp. 13-18, Prague, Czech Republic.

Hearst, M. (1998). WordNet: An electronic lexical database and some of its applications. In Fellbaum, C., editor, Automated Discovery of WordNet Relations. MIT Press.

Hearst, M. A. (1992). Automatic acquisition of hyponyms from large text corpora. In Proceedings of the 14th International Conference on Computational Linguistics, pages 539-545.

Hendrickx I., Morante R., Sporleder C., Antal v. d. Bosch (2002). ILK: Machine learning of semantic relations with shallow features and almost no data. Proceedings of the 4th International Workshop on Semantic Evaluations (SemEval-2007), pages 121124, Prague, June 2007

Jose Camacho-Collados, Mohammad Taher Pilehvar ' and Roberto Navigli (205). NASARI: a Novel Approach to a Semantically-Aware Representation of Items, Human Language Technologies. The 2015
Annual Conference of the North American Chapter of the ACL, pp 567-577, Denver, Colorado,USA.

Keet, C.M. and Artale, A. (2008). Representing and Reasoning over a Taxonomy of Part-Whole Relations. Applied Ontology, 2008, 3(1-2): 91-110

Marneffe M., MacCartney B. and Christopher D. Manning. (2006). Generating Typed Dependency Parses from Phrase Structure Parses. In LREC 2006.

Marshman, E. and M.-C. L' Homme. (2006).Disambiguation of lexical markers of cause and effect" In Picht, H. (ed.). Modern Approaches to Terminological Theories and Applications. Proceedings of the 15th European Symposium on Language for Special Purposes, LSP 2005. 261-285. Bern: Peter Lang.

Moldovan D., Badulescu A., Tatu M., Antohe D., and Girju R. (2004). Models for the semantic classification of noun phrases. In Proc. of the HLTNAACL 2004 Workshop on Computational Lexical Semantics, pages 60-67, Boston, USA.

Nakayama, K., Hara, T., and Nishio S. (2007). Wikipedia Mining for an Association Web Thesaurus Construction. In: Web Information Systems Engineering - WISE, Lecture Notes in Computer Science, Springer Berlin / Heidelberg, 322-334

Nakayama K., Hara T. and Nishio S. (2008). Wikipedia Link Structure and Text Mining for Semantic Relation Extraction. SemSearch 2008, CEUR Workshop Proceedings, ISSN 1613-0073, online at CEURWS.org/Vol-334/

Peter D. Turney and Michael L. Littman. (2005). Corpusbased learning of analogies and semantic rela-tions. Machine Learning, in press.

Peter D. Turney and Michael L. Littman. (2005). Corpus based learning of analogies and semantic relations. Machine Learning, 60(1-3):251-278

Peter D. Turney. (2006). Expressing implicit semantic relations without supervision. In Proceedings of ACL-2006.

Pribbenow, S. (1995). Parts and Wholes and their Relations. Habel, C. \& Rickheit, G. (eds.): Mental Models in Discourse Processing and Problem Solving. John Benjamins Publishing Company, Amsterdam

Strube, M., and Ponzetto, S.P. (2006). WikiRelate! Computing semantic relatedness using Wikipedia. In: Proceedings of the National Conference on Artificial Intelligence, pp. 1419- 1429

Taher M. and Navigli R. (2015). Align, Disambiguate and Walk: A Unified Approach for Measuring Semantic Similarity. Proceedings of the 51st Annual Meeting of the Association for Computational Linguistics, , Sofia, Bulgaria, 1341-1351, 
Winston, M., Chaffin, R. \& Hermann, D. (1987). Taxonomy of part-whole relations. Cognitive Science, 11(4), 417-444.

Yan Y., Matsuo Y. , Ishizuka M. (2009). An Integrated Approach for Relation Extraction from Wikipedia Texts . CAW2.0 2009, Madrid, Spain. 